\title{
Local Morphology Predicts Functional Organization of the Dorsal Premotor Region in the Human Brain
}

\author{
Céline Amiez, Penelope Kostopoulos, Anne-Sophie Champod, and Michael Petrides \\ Cognitive Neuroscience Unit, Montreal Neurological Institute, McGill University, Montreal, Quebec, Canada H3A 2B4
}

A confusing picture of the functional organization of the dorsal premotor region of the human brain emerged when functional neuroimaging studies that either examined visuomotor hand conditional activity or attempted to localize the human frontal eye field reported activity increases at the same general location, namely the junction of the superior precentral sulcus with the superior frontal sulcus. The present functional magnetic resonance imaging study examined visuomotor hand conditional activity and the locus of the frontal eye field as defined by a standard task, on a subject-by-subject basis, to clarify their location and reveal relationships between the pattern of local morphology and functional activity. The results demonstrate that visuomotor hand conditional activity and the frontal eye field lie within distinct parts of the superior precentral sulcus, revealing an organization of the human premotor cortex consistent with that observed in experimental studies in the monkey.

Key words: fMRI; dorsal premotor cortex; human; saccades; superior precentral sulcus; visuomotor conditional task

\section{Introduction}

The selection among multiple competing hand/arm motor acts on the basis of learned arbitrary conditional relationships to sensory cues, namely conditional associative motor responses, depends critically on the rostral part of the dorsal premotor cortex (Petrides, 2005). Lesions involving this region impair severely the learning and performance of visuomotor hand/arm conditional responses in both human subjects (Petrides, 1985, 1997; Halsband and Freund, 1990) and the monkey (Halsband and Passingham, 1982; Petrides, 1982, 1986). In addition, functional neuroimaging in normal human subjects has shown reliable activation related to the performance of visuomotor hand/arm conditional responses within the dorsal premotor cortex at the junction of the superior precentral sulcus (SPS) with the superior frontal sulcus (SFS) (Deiber et al., 1997; Grafton et al., 1998; Toni et al., 1999; Kurata et al., 2000). However, on the basis of functional neuroimaging studies, this same general region has also been reported to be the locus of the human homolog of the frontal eye field (FEF) (Paus, 1996; Petit et al., 1997; Corbetta et al., 1998; Luna et al., 1998; Petit and Haxby, 1999; Gagnon et al., 2002; Astafiev et al., 2003; Koyama et al., 2004; Grosbras et al., 2005). These functional neuroimaging findings raise a major problem regarding our understanding of the anatomo-functional organization of the dorsal premotor cortex in the human brain. How can the locus for visuomotor hand/arm conditional responses be the same as the locus of the FEF?

\footnotetext{
Received Nov. 4, 2005; revised Jan. 23, 2006; accepted Jan. 24, 2006.

This work was supported by Canadian Institutes of Health Research Grant FRN 37753 to M.P. C.A. was supported by the Fyssen Foundation. We thank Emily Rubin-Ferreira for technical help.

Correspondence should be addressed to Dr. Céline Amiez, Cognitive Neuroscience Unit, Montreal Neurological Institute, McGill University, 3801 University Street, Montreal, Quebec, Canada H3A 2B4. E-mail: camiez@bic.mni.mcgill.ca.

DOI:10.1523/JNEUROSCI.4739-05.2006

Copyright $\odot 2006$ Society for Neuroscience $\quad$ 0270-6474/06/262724-08\$15.00/0
}

The above conclusion is contrary to findings from experimental studies in monkeys that provide precise information about the organization of both the visuomotor hand/arm conditional region of the dorsal premotor cortex and the FEF in the caudal lateral frontal cortex (Bruce and Goldberg, 1985; Bruce et al., 1985; Schall, 1997; Tehovnik et al., 2000; Rizzolatti and Luppino, 2001; Dum and Strick, 2002). In the monkey, these two functional loci are known to be close to each other but clearly distinct. The dorsal premotor region that is critical for visuomotor hand/ arm conditional performance lies dorsal and caudal to the FEF (Halsband and Passingham, 1982; Petrides, 1982, 1986). Thus, assuming that the organization of these areas in the human brain is comparable to that of the monkey, we hypothesized that the focus of visuomotor hand conditional activity in the human would lie adjacent but dorsocaudal to the FEF close to the intersection of the SPS with the SFS.

The goal of the present functional magnetic resonance imaging (fMRI) experiment was to test the hypothesis that the organization of the dorsal premotor cortex in the human brain would be comparable to that of the monkey. Furthermore, we examined the data on a subject-by-subject basis because we wanted to reveal any relationship that may exist between functional activity and local morphological variations.

\section{Materials and Methods \\ Subjects}

Eight healthy volunteers (six females and two males) participated in this study (mean age, 28 years; range, 22-42 years). All subjects were righthanded as assessed by a handedness questionnaire (Crovitz and Zener, 1962). Informed, written consent was obtained from all of the participants according to the institutional guidelines established by the Ethics Committee of the Montreal Neurological Hospital and Institute.

\section{Experimental design}

The experimental question dictated the selection of the tasks. Because we wanted to examine the anatomo-functional relationships between the 
a. Visuo-motor hand conditional paradigm

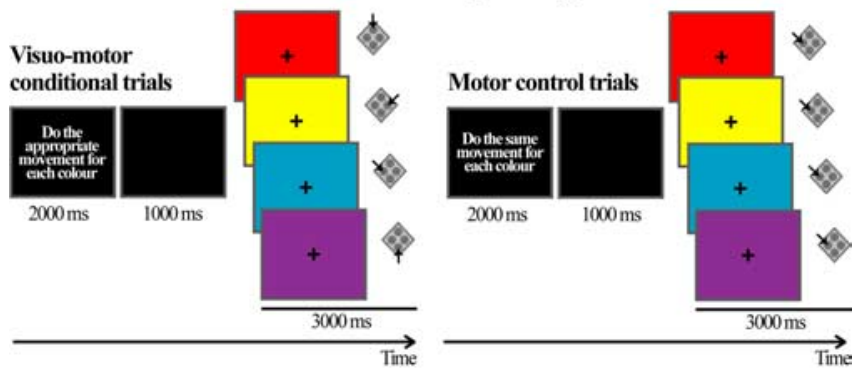

b. Saccadic eye movement paradigm

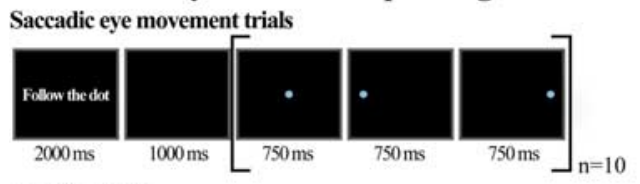

Fixation trials

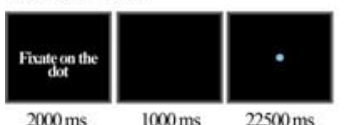

$2000 \mathrm{~ms}$

$1000 \mathrm{~ms}$

$2500 \mathrm{~ms}$

Figure 1. Behavioral tasks. $\boldsymbol{a}$, Visuomotor hand conditional task and motor control task. In both the visuomotor hand conditional and the motor control trials, one of four different colors was presented in a pseudo-random order in successive trials. The color occupied the entire screen. The subjects were instructed to look at the cross in the center of the colored screen during all trials. A written sentence at the beginning of each block of 16 trials indicated to the subjects the type of trial to be performed during that block. The sentence "Do the appropriate movement for each color" instructed the subjects that a block of 16 visuomotor hand conditional trials would follow. During these trials, the subjects had to press the appropriate one of four buttons in response to the presentation of one of the four colors. The arrows indicate the correct button to press depending on the presented color. The subjects were asked to press the top, left, right, and bottom buttons with their middle, index, and ring fingers and thumb, respectively. The sentence "Do the same movement for each color" instructed the subjects that a block of 16 motor control trials would follow. During these trials, the subjects had to press the same mouse button (with their index finger) for all of the colors presented. The duration of the intertrial interval was $1 \mathrm{~s} . \boldsymbol{b}$, Saccadic eye movement task and ocular fixation control task. The sentences "Follow the dot" and "Fixate on the dot" instructed the subject to perform saccadic eye movements or to fixate, respectively. During the saccadic eye movement trials, a dot was presented in one of three possible locations on the screen (i.e., left, center or right) for $750 \mathrm{~ms}$ in each location for a total of $22.5 \mathrm{~s}$. The subjects had to perform a saccade to follow the dot to its current location on the screen. During the ocular fixation trials, the subjects had to fixate on the dot presented in the center of the screen for $22.5 \mathrm{~s}$.

dorsal premotor region involved in visuomotor hand conditional activity and the FEF, we used a standard saccadic eye movement task to localize the human FEF and a standard task to localize visuomotor hand conditional activity. Subjects performed the visuomotor hand conditional task and the saccadic eye movement task during successive blocks of trials in the present block-design fMRI study. Stimulus presentation and the recording of motor responses were computer controlled and programmed with E-prime 1.1 (Psychology Software Tools, Pittsburgh, PA).

Visuomotor hand conditional task and motor control task. These tasks were designed to examine changes in activity specific to the performance of well learned visuomotor hand conditional responses. A written instruction on the screen at the beginning of each block of trials informed the subjects of the type of the trial that would follow. The sentence "Do the appropriate movement for each color" instructed the subjects that a block of 16 visuomotor hand conditional trials would follow. In this block of trials, four different colors that occupied the entire screen were presented randomly on successive trials of $3 \mathrm{~s}$ duration with a $1 \mathrm{~s}$ intertrial interval. The subjects had to press the appropriate one of four buttons on a computer mouse in response to the presentation of each one of the different colors (red, yellow, blue, and purple) (Fig. 1). The sentence "Do control movement" instructed the subjects that a block of 16 motor control trials would follow. In this block of trials, the same four different colors were presented randomly one at a time on successive trials of $3 \mathrm{~s}$ duration each and with a $1 \mathrm{~s}$ intertrial interval. The subjects had to press the same mouse button (with their index finger) for all colors presented. While performing both tasks, subjects were instructed to maintain ocular fixation on the cross presented in the center of the screen.

One day before the scanning session, all participants learned to perform these tasks outside the scanner until they reached a level of $95 \%$ correct responses on two consecutive sets of 32 trials.

Saccadic eye movement task and ocular fixation control task. These tasks were designed to examine changes in activity specific to the performance of saccadic eye movements. For the saccadic eye movement task, we used the one described by Gagnon et al. (2002). The sentence "Follow the dot" instructed the subjects that they would have to perform saccadic eye movements. In this task, a dot was successively and randomly presented in one of three possible locations on the screen (i.e., left, center, right) (Fig. 1) for $750 \mathrm{~ms}$ in each location for a total duration of $22.5 \mathrm{~s}$. The subjects had to perform a saccade to follow the dot to its current location on the screen. The sentence "Fixate on the dot" instructed the subjects that they would have to keep looking at the dot located in the center of the screen for $22.5 \mathrm{~s}$.

\section{MRI acquisition}

Scanning was performed on a 1.5 T Sonata MRI Scanner (Siemens, Erlangen, Germany). Because of the loud "pinging" noise of the functional multislice echo-planar imaging, subjects wore earplugs. After a highresolution $\mathrm{T} 1$ anatomical scan (entire head, $1 \mathrm{~mm}^{3}$ isotropic resolution), five or six runs of 120 images each [ 38 oblique T2* gradient echo-planar images; voxel size, $3.4 \times 3.4 \times 3.4 \mathrm{~mm}$; repetition time (TR), $3.5 \mathrm{~s}$; echo time, $45 \mathrm{~ms}$; flip angle, $90^{\circ}$ ] sensitive to the blood oxygenation leveldependent (BOLD) signal were acquired. Visual stimuli were presented through a liquid crystal display projector with a mirror system, and the responses of the subjects were recorded with an MR-compatible optical computer mouse. Each subject was required to complete five or six runs of fMRI data collection during the scanning session, each one lasting $\sim 7.5 \mathrm{~min}$. In each run, the subjects performed twice the following sequence of blocks of trials: 16 visuomotor hand/arm conditional trials, 16 hand/arm motor control trials, ocular fixation for $22.5 \mathrm{~s}$, and saccadic eye movements for $22.5 \mathrm{~s}$. The first trial of each block of trials was synchronized with the scanner acquisition via a trigger signal generated by the scanner. Behavioral and imaging data were acquired for all trials.

\section{Data analysis}

Contrasts analyzed. To establish the brain regions involved in the performance of well learned conditional motor responses of the hand, we compared the BOLD signal of the visuomotor hand conditional task with that of the motor control task. To assess the brain regions involved in the performance of saccadic eye movements, we compared the BOLD signal obtained during the saccadic eye movement task with that obtained during the ocular fixation task. To establish the location of the hand region of the primary motor cortex, we compared the BOLD signal obtained in the motor control task with that of the ocular fixation task.

Statistical analysis. The first three volumes of each functional run were discarded because of the T1 saturation effects. Medical Image NetCDF (MINC) images from all runs were first realigned with an AFNI image registration software using the third frame of the first run as a reference (Cox and Jesmanowicz, 1999) and smoothed with an MINC blurring software (mincblur) using a $6 \mathrm{~mm}$ full-width half-maximum (FWHM) isotropic Gaussian kernel. Subsequently, all images were transformed into standard proportional stereotaxic space (Talairach and Tournoux, 1988) using in-house dedicated software (Collins et al., 1994). Functional and anatomical data in each individual subject were then merged to locate regions of significant activation.

The data analysis was performed using fmristat (Worsley et al., 2002) (available at http://www.math.mcgill.ca/keith/fmristat). The statistical analysis of the fMRI data was based on a linear model with correlated errors. The design matrix of the linear model was first convolved with a hemodynamic response function modeled as a difference of two gamma functions timed to coincide with the acquisition of each slice (Glover, 1999). Drift was removed by adding cubic spline covariates in the frame times, one covariate per 2 min of scanning, to the design matrix (Worsley et al., 2002). 
Sufficient sampling across the hemodynamic response function was acquired in this experiment because of the complete desynchronization of the trial onset time from the acquisition repetition time. This desynchronization was achieved by having a fixed repetition time of acquisition (TR, $3.5 \mathrm{~s}$ ) and a total trial duration that lasted $4 \mathrm{~s}$ in visuomotor hand/arm conditional and motor control trials, $22.5 \mathrm{~s}$ in saccadic eye movement trials, and $22.5 \mathrm{~s}$ in ocular fixation trials. The correlation structure was modeled as an autoregressive process of degree 1 (Bullmore et al., 1996). At each voxel, the autocorrelation parameter was estimated from the least-squares residuals using the Yule-Walker equations, after a bias correction for correlations induced by the linear model. The autocorrelation parameter was first regularized by spatial smoothing with a $15 \mathrm{~mm}$ FWHM Gaussian filter and then used to "whiten" the data and the design matrix. The linear model was then reestimated using least squares on the whitened data to produce estimates of effects and their SEs (Worsley et al., 2002).

In a second step, experimental runs within a subject were combined with a fixed-effects analysis that involved estimating the ratio of the random-effects variance to the fixed-effects variance, then smoothing this ratio with an infinitely large FWHM Gaussian filter, yielding a global ratio of 1 . The variance of the effect was then estimated by the smoothed ratio multiplied by the fixed-effects variance to achieve higher degrees of freedom (Worsley et al., 2002).

The resulting $t$ statistic images were thresholded using the minimum given by a Bonferroni's correction and random field theory, taking into account the non-isotropic spatial correlation of the errors. Significance was assessed on the basis of the spatial extent of consecutive voxels.

A cluster volume extent $>175 \mathrm{~mm}^{3}$, associated with a $t$ value $>3$, was significant $(p<$ 0.05 ), corrected for multiple comparisons using the method of Friston et al. (1995). For a single voxel in an exploratory search involving all peaks within an estimated gray matter of 600 $\mathrm{cm}^{3}$ covered by the slices, the threshold for reporting a peak as significant $(p<0.05)$ was $t=$ 4.118 (Worsley et al., 1996).

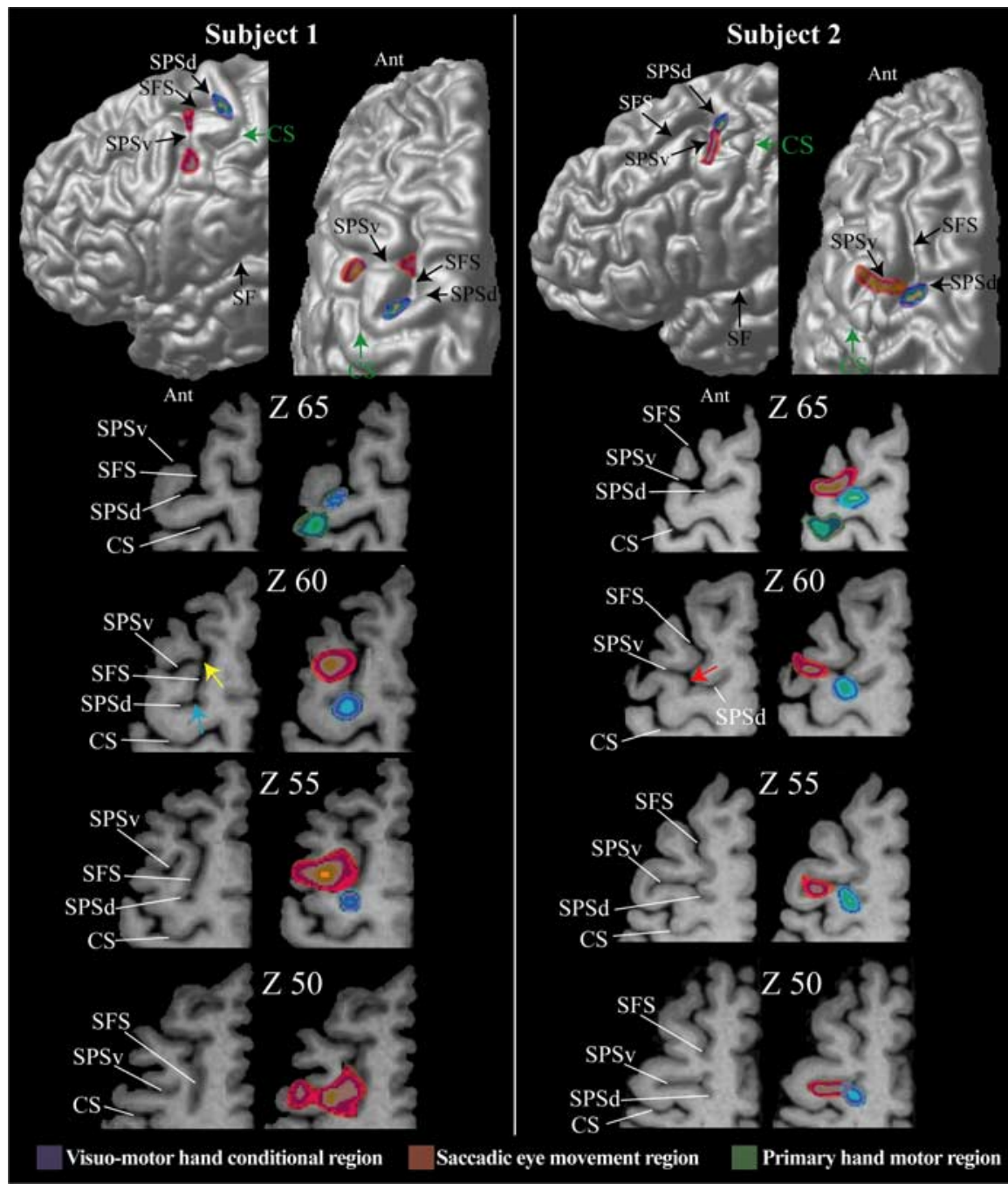

Figure 2. The location of the premotor hand region for conditional motor responses is in blue, the location of the saccadic eye movement region (i.e., the FEF) is in red, and the location of the primary motor cortex hand representation is in green in subjects 1 and 2. The foci of activity illustrated result from the subtractions reported in Tables 1-3. These foci are shown on each subject's left hemisphere: lateral view (top left diagram) and top view (top right diagram). The green arrow indicates the point of the central sulcus in the depth of which the primary hand motor representation is located (i.e., precentral knob). For each subject, horizontal sections at different levels ( $z$ coordinate) in standard stereotaxic space are shown. The left horizontal sections are anatomical MRIs, and the right sections are the same ones with the premotor hand conditional (blue) and saccadic eye movement (red) foci displayed. In both subjects, the primary hand motor region is also displayed in green. In subject 1, the blue arrow marks the point of intersection of the SPSd with the caudal end of the SFS, and the yellow arrow marks the point of intersection of the SPSV with the SFS. In subject 2, the red arrow indicates the common point of intersection of the SPSd, the SPSv, and the caudal end of the SFS. Ant, Anterior part of the brain; CS, central sulcus; SF, Sylvian fissure.

\section{Results}

Morphological variation in the dorsal premotor region

The most significant morphological feature of the dorsal premotor region of the human brain is the junction of the SPS with the caudal end of the SFS. Detailed examination of the morphology of this region in coronal, sagittal, and horizontal sections shows that the SPS is not a single sulcus but rather a complex of two distinct sulci that often blend together close to the caudal end of the SFS. Thus, on the surface of the brain, these two sulci give the impression of one continuous dorsoventrally directed SPS [subject 2 (Fig. 2), subjects 3 and 5 (Fig. 3), and subject 8 (Fig. 4)]. Because in these cases (Fig. $5 c$, type 1), there is a common point of intersection with the caudal end of the SPS, we can speak of a dorsal branch of the SPS (SPSd) that lies above the point of intersection and a ventral branch (SPSv) that lies below this point. For example, this morphological relationship can be clearly ap- preciated in horizontal section Z60 of subject 2 in Figure 2, in which the red arrow indicates this common point of intersection of the SPSd, the SPSv, and the caudal end of the SFS. However, in many other brains (Fig. $5 c$, type 2a), these three sulci do not meet at the same point (Germann et al., 2005). In these cases, the SPSv joins the SFS anterior to the point of intersection of the SPSd with the caudal end of the SFS [subject 1 (Fig. 2), subject 6 (Fig. 3), and subject 7 (Fig. 4)]. As an example, see horizontal section Z60 of subject 1 in Figure 2, in which the blue arrow marks the point of intersection of the SPSd with the caudal end of the SFS and the yellow arrow marks the point of intersection of the SPSv with the SFS. Indeed, in a few cases, the SPSv does not intersect with the caudal end of the SFS and remains clearly separated from it by a narrow gyral passage [asterisk in subject 7 (Fig. 4) and type 2b (Fig. 5c)].

The SPS runs more or less parallel to the superior part of the 


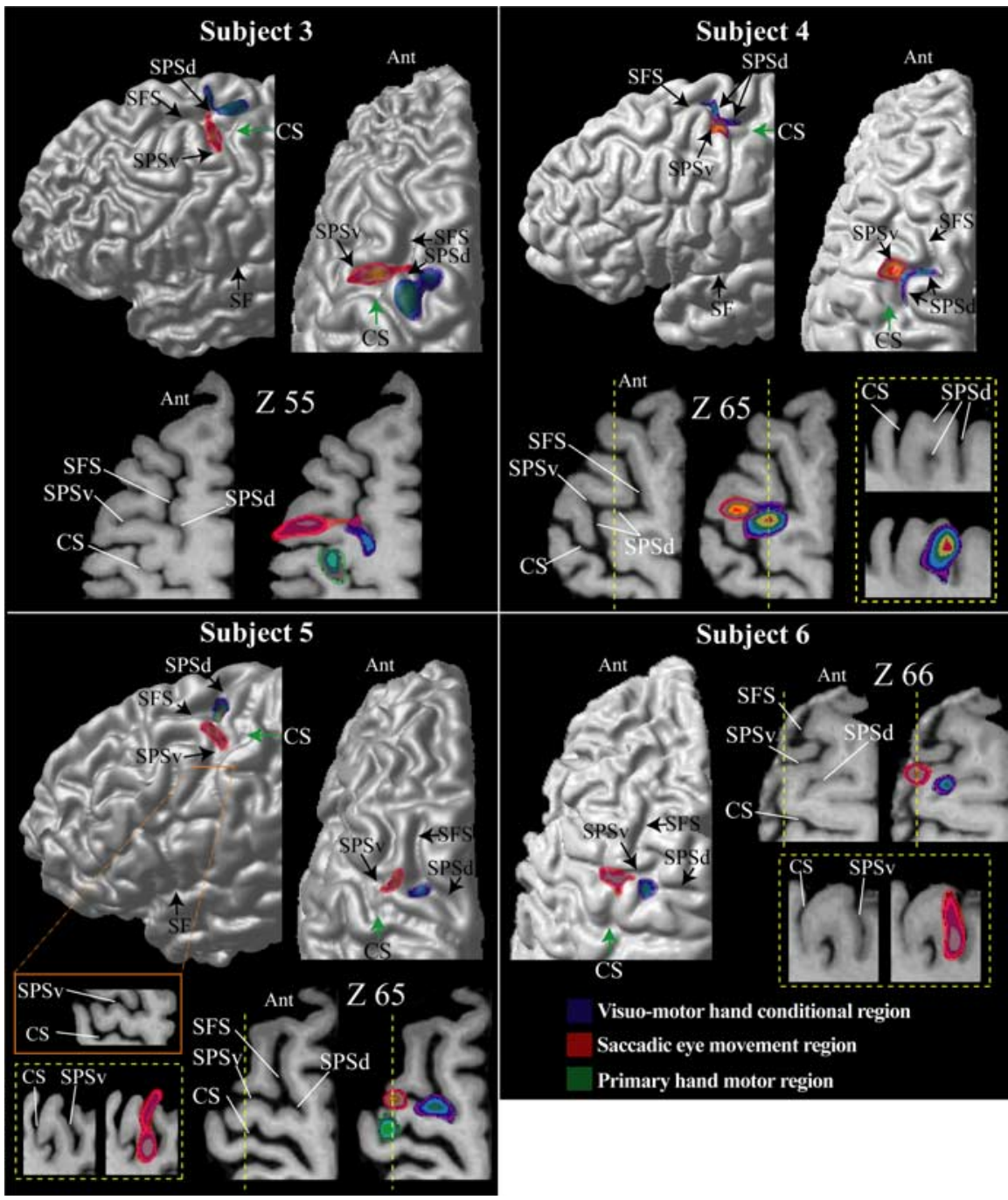

Figure 3. The location of the premotor hand region for conditional motor responses is in blue, the location of the saccadic eye movement region (i.e., the FEF) is in red, and the location of the primary motor cortex hand representation is in green in subjects 3-6. The foci of activity illustrated result from the subtractions reported in Tables $1-3$. These foci are shown on each subject's left hemisphere: lateral view (top left diagram) and top view (top right diagram). The green arrow indicates the point of the central sulcus in the depth of which the primary hand motor representation is located (i.e., Broca's pli de passage moyen). For each subject, horizontal sections at different levels (z coordinate) in standard stereotaxic space are shown. The left horizontal sections are anatomical MRIs, and the right sections are the same ones with the premotor hand conditional (blue) and saccadic eye movement (red) foci displayed. In subjects 3 and 5, the primary hand motor region is also displayed in green. In subjects 4-6, the yellow dotted line indicates the level of the sagittal section (i.e., the $x$ coordinate) illustrated within the yellow dotted box. Note that, in subject 5, the precentral gyrus has receded and therefore the SPSv and the central sulcus blend in certain locations. This can be appreciated by careful inspection of the horizontal section indicated by the orange line and box. Ant, Anterior part of the brain CS, central sulcus; SF, Sylvian fissure.

central sulcus, thus forming the anterior border of the precentral gyrus. Normally, the SPSd is oriented toward the midline of the brain. However, in one of the subjects we studied (Fig. 3, subject 4), the SPSd curved sharply posteriorly toward the central sulcus and blended with it. As a result, the point of intersection between the SPSd and the SFS in this subject was much more anterior than in all the other brains.

Despite these considerable variations in the local morphology of the region of interest, a subject-by-subject analysis of the locus of functional activity revealed a clear relationship between morphology and functional activity (see below). Because the purpose of the present study was to test a specific hypothesis about the organization of the dorsal premotor cortex in the human brain, the detailed subject-by-subject analysis is focused on activity changes within the dorsal premotor region related to performance in our two tasks, namely the visuomotor hand conditional and the saccadic eye movement tasks.

\section{Visuomotor hand conditional activity and the SPSd}

In the visuomotor hand conditional task, the subjects had to select and perform the appropriate one from a set of four actions (i.e., button presses with the middle, index, or ring finger, or thumb) on the basis of well learned arbitrary associations to visual stimuli (red, yellow, blue, and purple colors). To isolate activity specific to the performance of the conditional hand motor responses, we compared activity in the visuomotor hand conditional task with that in the motor control task. We computed $t$ statistical maps of the comparison (visuomotor hand conditional task minus motor control task) in each individual subject, and we superimposed these maps on the anatomical MRI of each individual subject. A significant increase in activity was consistently observed in the left dorsal premotor cortex in each individual subject (Figs. 2-4). The focus of this activity increase was in the dorsal branch of the SPS close to the caudal end of the SFS in the left hemisphere in each subject [average Montreal Neurological Institute (MNI) coordinates $(x, y, z):-25.8 \pm 3.7 \mathrm{SD},-14.4 \pm$ 4.5 SD, $61.5 \pm 8.4$ SD] (Table 1). A similar significant increase in activity in the dorsal branch of the SPS close to the junction with the SFS was observed in the right hemisphere, but only in six of the eight subjects [average MNI coordinates $(x, y$, $z): 30.3 \pm 5.7 \mathrm{SD},-17.4 \pm 4 \mathrm{SD}, 64 \pm 6.3$ $\mathrm{SD}]$. It is important to note that no activity increase was found in any part of the lateral prefrontal cortex. Note also that, in the left hemisphere, significant increases in activity were also observed in the supplementary motor area [average MNI coordinates $(x, y, z)$, left hemisphere: $-3.5 \pm$ $2.5 \mathrm{SD},-6.5 \pm 7.3 \mathrm{SD}, 53.5 \pm 2.7 \mathrm{SD}]$, the ventral premotor cortex [average MNI coordinates $(x, y, z)$, left hemisphere: $-53 \pm 6.9 \mathrm{SD},-0.4 \pm 11.2$ $\mathrm{SD}, 42.8 \pm 7 \mathrm{SD}$ ], and the parietal cortex [average MNI coordinates $(x, y, z)$ : left hemisphere, $-41.3 \pm 5.6 \mathrm{SD},-38.3 \pm 5.1 \mathrm{SD}$, $53 \pm 10$ SD (first peak); $-25.4 \pm 4.3 \mathrm{SD},-62.5 \pm 5.7 \mathrm{SD}, 58.8 \pm$ 8.4 SD (second peak); right hemisphere, $30.2 \pm 5.7 \mathrm{SD},-55.7 \pm$ 3.4 SD, $65.3 \pm 4.2 \mathrm{SD}$ ] (data not shown).

Primary motor cortex hand activity in the central sulcus To determine the location of the hand representation within the primary motor cortical region in the central sulcus (area 4), we compared activity during the motor control task to the central ocular fixation task. This comparison revealed a significant increase in activity within the known locus of the primary motor hand representation in the central sulcus, namely the "precentral 
knob" (Yousry et al., 1997), also known as Broca's pli de passage moyen (Broca, 1888; Boling et al., 1999), in the left hemisphere in each individual subject [average MNI coordinates $(x, y, z):-37.1 \pm 6.7 \mathrm{SD}$, $-25.6 \pm 5.4 \mathrm{SD}, 62.5 \pm 5 \mathrm{SD}$ ] (Figs. 2-4, $5 d$; Table 2). Because the subjects were responding with their right hand, the increase in activity was within the primary motor cortical region of the left hemisphere.

\section{Activity related to saccadic eye movements and the SPSv}

The comparison of cerebral activity during the saccadic eye movement task with that in the ocular fixation task was used to determine the location of activity related to saccadic eye movement within the caudal lateral frontal lobe (i.e., the FEF). This comparison revealed a specific increase in activity within the ventral branch of the SPS in both the left and the right hemispheres of each individual subject (Table 3 , Figs. 2-4) [average MNI coordinates ( $x, y$, $z)$ : left hemisphere, $-38 \pm 4.2 \mathrm{SD},-9.8 \pm$ $3.8 \mathrm{SD}, 54.4 \pm 7.5 \mathrm{SD}$; right hemisphere, $34.5 \pm 9.1 \mathrm{SD},-10.8 \pm 7.7 \mathrm{SD}, 52.5 \pm 5.3$ SD]. Importantly, in all subjects, the $t$ statistic within the dorsal branch of the SPS was dramatically lower in the comparison "saccadic eye movement task minus the ocular fixation task" (i.e., average $t$ statistic $=2.09 \pm 2.22$ ) than in the comparison "visuomotor hand conditional task minus motor control task" (i.e., average $t$ statistic $=6.24 \pm 2.51)$.

Note that an additional peak of activity was observed in the dorsal part of the inferior precentral sulcus [average MNI coordinates $(x, y, z)$, left hemisphere: $-48 \pm 7$ $\mathrm{SD},-6.6 \pm 5.8 \mathrm{SD}, 45.4 \pm 6.5 \mathrm{SD}]$, the supplementary eye field [average MNI coordinates $(x, y, z)$, left hemisphere: $-4.8 \pm$ $2.8 \mathrm{SD},-9 \pm 5.3 \mathrm{SD}, 70.5 \pm 3.8 \mathrm{SD}]$, the supplementary motor area [average MNI coordinates $(x, y, z)$, left hemisphere: $-5.1 \pm 4.3 \mathrm{SD},-2.6 \pm 6.4 \mathrm{SD}, 55.5 \pm 3.7$ $\mathrm{SD}$ ], and in the parietal cortex [average MNI coordinates $(x, y, z)$ : left hemisphere, $-32.4 \pm 6.1 \mathrm{SD},-54.8 \pm 6.1 \mathrm{SD}, 50.9 \pm$ $6.8 \mathrm{SD}$; right hemisphere, $32 \pm 6.2 \mathrm{SD}$, $-53.6 \pm 6.6 \mathrm{SD}, 52.1 \pm 8.9 \mathrm{SD}]$.

In a recent study, Blanke et al. (2000), using electrical stimulation, reported evoked saccadic eye movements in three patients in the posterior-most part of the middle frontal gyrus, just anterior to the junction of the SPS and below the SFS. Our placement of the FEF in relation to the ventral part of the SPS, which is either located at the very end of the middle frontal gyrus (Fig. $5 c$, type 1 ) or cuts into the most

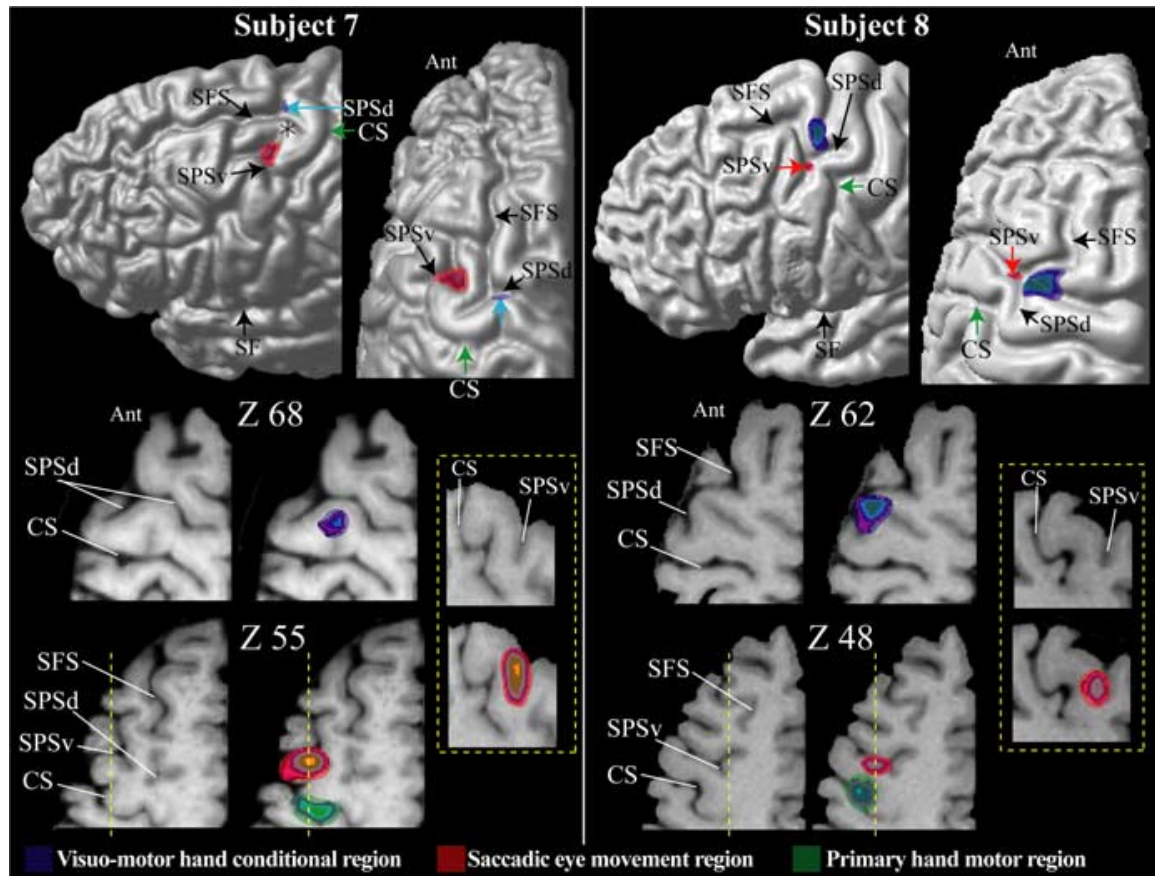

Figure 4. The location of the premotor hand region for conditional motor responses is in blue, the location of the saccadic eye movement region (i.e., theFEF) is in red, and the location of the primary motor cortex hand representation is in green in subjects 7 and 8 . The foci of activity illustrated result from the subtractions reported in Tables 1-3. These foci are shown on each subject's left hemisphere: lateral view (top left diagram) and top view (top right diagram). The green arrow indicates the point of the central sulcus in the depth of which the primary hand motor representation is located (i.e., Broca's pli de passage moyen). For each subject, horizontal sections at different levels ( $z$ coordinate) in standard stereotaxic space are shown. The left horizontal sections are anatomical MRIs, and the right sections are the same ones with the premotor hand conditional (blue) and saccadic eye movement (red) foci displayed. In both subjects, the primary hand motor region is also displayed in green. In both subjects, the yellow dotted line indicates the level of the sagittal section (i.e., the $x$ coordinate) illustrated within the yellow dotted box. Note that (1) on the lateral and top views of subject 8, the red arrow points to the location of the SPSv in the depth of which the saccadic eye movement region is located; (2) on the lateral and top views of subject 7, the blue arrow points to the location of the SPSd in the depth of which the premotor hand region for motor conditional responses is located; and (3) in the top view of subject 7, the asterisk indicates the narrow gyral passage between the SPSd and the SPSv. Ant, Anterior part of the brain; CS, central sulcus; SF, Sylvian fissure.

Table 1. Localization of the visuomotor hand conditional region: visuomotor hand conditional task minus motor control task

\begin{tabular}{|c|c|c|c|c|}
\hline \multirow[b]{2}{*}{ SPSd region } & \multicolumn{3}{|l|}{ MNI coordinates } & \multirow[b]{2}{*}{$t$ statist } \\
\hline & $x$ & $y$ & $z$ & \\
\hline \multicolumn{5}{|l|}{ Left hemisphere } \\
\hline \multirow[t]{2}{*}{ Subject 1} & -28 & -18 & 72 & 9.51 \\
\hline & -28 & -22 & 60 & 5.55 \\
\hline \multirow[t]{2}{*}{ Subject 2} & -28 & -10 & 62 & 7.79 \\
\hline & -28 & -10 & 50 & 5.06 \\
\hline \multirow[t]{2}{*}{ Subject 3} & -28 & -16 & 70 & 11.18 \\
\hline & -22 & -14 & 48 & 5.60 \\
\hline Subject 4 & -22 & -14 & 64 & 6.81 \\
\hline Subject 5 & -20 & -18 & 66 & 6.51 \\
\hline Subject 6 & -26 & -14 & 51 & 3.84 \\
\hline Subject 7 & -22 & -16 & 68 & 2.71 \\
\hline Subject 8 & -32 & -6 & 66 & 4.04 \\
\hline Mean \pm SD & $-25.8 \pm 3.7$ & $-14.4 \pm 4.5$ & $61.5 \pm 8.4$ & \\
\hline \multicolumn{5}{|c|}{ Right hemisphere } \\
\hline Subject 1 & 32 & -20 & 64 & 5.34 \\
\hline Subject 2 & 38 & -18 & 68 & 8.10 \\
\hline \multirow[t]{2}{*}{ Subject 3} & 32 & -22 & 68 & 5.95 \\
\hline & 28 & -20 & 58 & 4.50 \\
\hline \multicolumn{5}{|l|}{ Subject 4} \\
\hline Subject 5 & 28 & -12 & 58 & 10.17 \\
\hline \multicolumn{5}{|l|}{ Subject 6} \\
\hline Subject 7 & 34 & -18 & 58 & 3.26 \\
\hline Subject 8 & 20 & -12 & 74 & 4.54 \\
\hline Mean $\pm S D$ & $30.3 \pm 5.7$ & $-17.4 \pm 4$ & $64 \pm 6.3$ & \\
\hline
\end{tabular}

Data are the maxima of regions showing significant increases in the BOLD signal in each individual subject. All $t$ statistics are significant at $p<0.05$ corrected for multiple comparisons. The stereotaxic coordinates are expressed in millimeters within the MNI stereotaxic proportional system that is based on the Talairach and Tournoux (1988) stereotaxic space: $x$, medial-to-lateral distance relative to the midline (positive, right); $y$, anterior-to-posterior distance relative to the anterior commissure (positive, anterior); $z$, superior-to-inferior distance relative to the anterior commissure-posterior commissure line (positive, superior). 


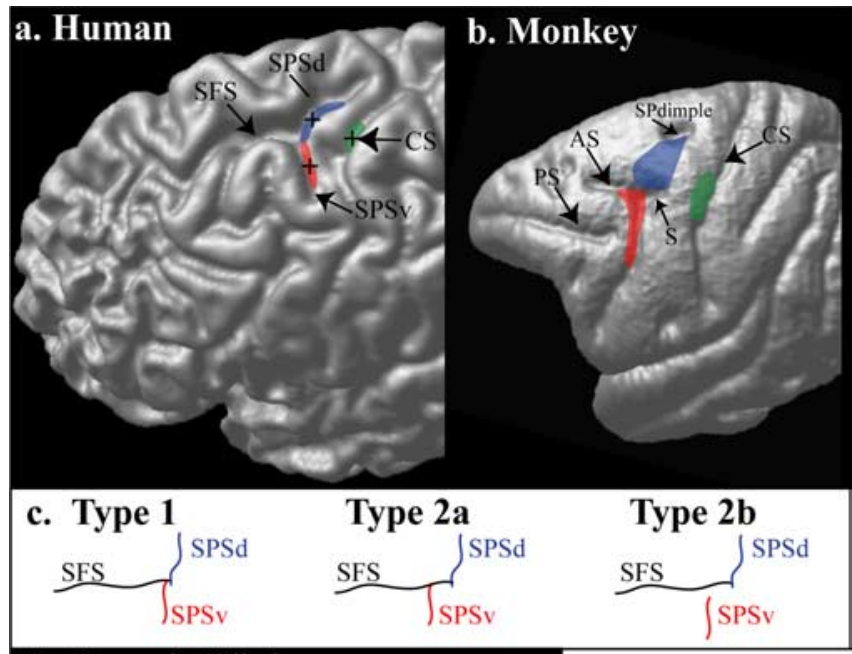

d. Broca's Pli de passage moyen

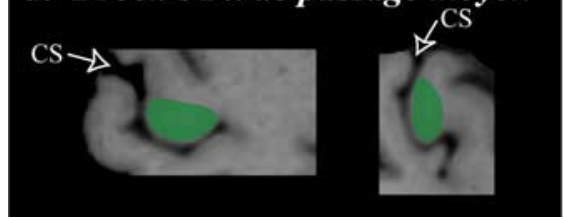

Figure 5. The premotor hand region, the saccadic eye movement region (i.e., the FEF), and the primary motor cortex hand representation in the human and monkey frontal cortex. $\boldsymbol{a}$, The premotor cortex hand region for conditional responses (blue), the saccadic eye movement region (red), and the primary motor cortex hand representation (green) are shown on the cortical surface of the three-dimensional rendering of the left hemisphere of one human brain in standard stereotaxic space. The crosses correspond to the mean locations of activities reported in Tables 1-3. The blue, red, and green regions correspond to an estimate of the size of these regions based on the subject-by-subject analysis. $\boldsymbol{b}, 0 \mathrm{n}$ the left hemisphere of a macaque monkey brain, the following are indicated: the location of the dorsal premotor region involved in the performance of hand conditional responses (blue); the saccadic eye movement region (i.e., the classical FEF) in the concavity of the arcuate sulcus (red); and the hand representation of the primary motor cortex (green). c, Schematic representation of the sulcal patterns in the dorsal premotor region of the human brain. Type 1, The SPSd and SPSV approach each other at the caudal end of the SFS, giving the impression of one continuous sulcus; type 2a, the SPSv joins the SFS at a more anterior location than the SPSd; type $2 b$, the SPSv approaches but does not join the SFS. $\boldsymbol{d}$, The hand representation of the primary motor cortex (area 4) in the human brain lies within the central sulcus at the level indicated in green in $\boldsymbol{a}$. Within the central sulcus, the hand representation occupies a distinct morphological feature, a fold known as the precentral knob. A horizontal (left) and a sagittal (right) section through this part of the central sulcus illustrate this distinct morphological feature. CS, Central sulcus; AS, arcuate sulcus; PS, principalis sulcus; S, spur; SPdimple, superior precentral dimple.

posterior part of the middle frontal gyrus (Fig. $5 c$, type 2), is more consistent with the results of Blanke et al. (2000) than previous studies that reported the intersection of the SFS with the SPS as the locus of the FEF. Of course, the current levels used in electrical stimulation studies will affect the observed extent of the FEF (i.e., the larger the stimulation current, the greater will be the extent of the area defined as the FEF). Thus, the greater anterior extent of the FEF as defined by Blanke et al. (2000) may be related to the stimulation currents that they used.

\section{Discussion}

Many previous functional neuroimaging studies (for review, see Grosbras et al., 2005) had reported that the intersection of the SPS with the caudal end of the SFS is the homolog of the FEF that had originally been established, on the basis of electrophysiological studies, in the monkey (Bruce and Goldberg, 1985; Bruce et al., 1985; Schall, 1997; Tehovnik et al., 2000). At the same time, other functional neuroimaging studies reported this same inter- section of the SPS with the SFS to be the focus of premotor hand/ arm conditional activity (Deiber et al., 1997; Grafton et al., 1998; Toni et al., 1999; Kurata et al., 2000). These functional neuroimaging findings raised a major problem regarding our understanding of the organization of the dorsal premotor region of the human brain by failing to discriminate between the location of visuomotor hand conditional activity and the FEF.

Experimental anatomical and functional studies in macaque monkeys had clearly shown that the FEF lies in the concavity of the arcuate sulcus (Bruce and Goldberg, 1985; Bruce et al., 1985; Schall, 1997; Tehovnik et al., 2000), ventral and slightly rostral to the hand/ arm premotor representation that has been shown to be critical for visuomotor hand conditional performance (Halsband and Passingham, 1982; Petrides, 1982, 1986; Mitz et al., 1991; Boussaoud and Wise, 1993a,b; Germain and Lamarre, 1993). Thus, the experimental data available from non-human primates strongly predicted that the FEF should lie ventral and slightly rostral to the locus of visuomotor hand conditional activity. In the present fMRI study, we tested this hypothesis by examining both visuomotor hand conditional activity and eye movement activity, on a subject-by-subject basis. The data showed that, in the human dorsal premotor region at the junction of the SPS with the SFS, the visuomotor hand conditional premotor locus of activity lies near, but always dorsocaudal, to the FEF, as would be predicted from anatomical and physiological studies in the macaque monkey (Fig. 5).

Importantly, our data demonstrate that the visuomotor hand conditional premotor activity and the saccadic eye movement activity are consistently related to particular aspects of the local morphology of the dorsal premotor region, so that one can predict the locus of functional activity from the local morphological pattern in a particular brain. The visuomotor hand conditional premotor activity was consistently located in the depth of the dorsal branch of the SPS, close to its intersection with the caudal end of the SFS. In contrast, the saccadic eye movement activity was consistently located in the depth of the ventral branch of the SPS. Furthermore, functional activity was closely related to two morphological variants of the linkage of the ventral branch of the SPS to the caudal SFS. In the first variant (type 1), the ventral branch of the SPS ends, dorsally, close to the caudal end of the SFS and therefore has a more or less common point of intersection with the dorsal branch of the SPS (Figs. 2-4, subjects 2, 3, 5, and 8 ). In these cases, in which the SPS has a more or less continuous course, the saccadic eye movement activity lies below the common point of intersection within the ventral branch of the SPS, whereas the visuomotor hand conditional activity lies at the point of intersection, extending dorsally within the dorsal branch of the SPS. In the second variant (type 2), the ventral branch of the SPS joins the SFS at a much more anterior point than the dorsal branch of the SPS (Figs. 2-4, subjects 1, 6 and 7). Thus, in this variant, there is no common point of intersection between the three sulci SPSd, SPSv, and SFS. Importantly, in these cases, the saccadic eye movement activity is located in the more anterior ventral branch of the SPS, whereas the visuomotor hand conditional functional activity remains at the point of intersection of the dorsal branch of the SPS with the SFS, extending dorsally (Fig. $5 a$ ). Thus, in all subjects examined, the locus of functional activity was tightly related to the local morphology.

Our observation that the visuomotor hand conditional premotor activity is located in the dorsal branch of the SPS is consistent with two recent studies reporting that trancranial magnetic stimulation of the dorsal part of the SPS induced interference with the performance of a visuomotor conditional task (Schluter et al., 1998; Johansen-Berg, 2002). 
Studies in the monkey demonstrated a striking dissociation between the effects of lesions to the rostral part of the dorsal premotor cortex and more anterior dorsolateral prefrontal lesions involving areas 9 and 46 (Petrides, 1982, 1986, 2005). Whereas lesions involving the rostral premotor cortex yield a massive impairment both in the learning and performance of motor conditional responses (Halsband and Passingham, 1982; Petrides, 1982, 1986, 2005), lesions of the dorsolateral prefrontal cortex yield either no impairment or a negligible one. The present fMRI data are consistent with the observations from the lesion studies in the monkey in so far as no activity increase was found in the dorsolateral prefrontal cortex during the performance of well learned visuomotor hand conditional responses.

A recent fMRI study (Boettiger and D'Esposito, 2005) compared frontal cortex activity during the learning, by trial and error, of novel arbitrary visuomotor response associations with the performance of well learned (i.e., familiar) responses. As would be expected, the comparison of novel learning with familiar well learned visuomotor responses yielded increased activity in several prefrontal areas, which is reflecting greater involvement of general purpose control processes (e.g., monitoring) during the learning of novel associations. Also, as would be expected, this comparison did not reveal any activity in the rostral part of the dorsal premotor cortex, because this region would be involved both in the execution of visuomotor conditional responses during the novel learning condition and also in the execution of familiar well learned visuomotor associations. A negative peak from this comparison (i.e., novel learning minus execution of familiar associations) in the superior frontal gyrus [MNI coordinates $(x, y, z):-6,38,58)$ was interpreted by the authors as "premotor" activity representing well learned rules. However, activity at $y=38$ lies approximately in the middle of the superior frontal gyrus where rostral areas $8 \mathrm{~B}$ and 9 are located and lies at least $50 \mathrm{~mm}$ anterior to the intersection of the SPS with the caudal end of the SFS where the visuomotor hand conditional premotor region (area 6) is represented (at $y=-14)$ (Table 1). We looked directly in our data set (visuomotor hand conditional task minus motor control task) at coordinates $(x, y, z)-6,38,58$ and found no evidence of increased activity (average $t$ value $=-0.56 \pm 1.1$ ) related to the performance of well learned visuomotor associations. We therefore suggest that the peak at coordinates $(x, y, z)-6,38,58$ in the above study represents decreased activity in the dorsal prefrontal cortical area 9 during the execution of familiar visuomotor acts compared with the novel learning of such acts that would inevitably place greater emphasis on monitoring and other executive processes known to be controlled by the dorsolateral prefrontal cortex (including area 9).

Finally, our data confirmed previous work that the represen- tation of the hand in the primary motor cortex (area 4) is located in the precentral knob (Broca, 1888; Yousry et al., 1997; Boling et al., 1999), which is found in the depth of the central sulcus. Note that the location of the premotor visuomotor hand conditional activity was anterior to the primary motor representation of the hand (indicated in green in all subjects in Figs. 2-4).

In conclusion, the organization of the human dorsal premotor region, as revealed in the present study, was remarkably consistent with predictions from experimental studies in the macaque monkey. As in the monkey (Halsband and Passingham, 1982; Petrides, 1982, 1986; Bruce and Goldberg, 1985; Bruce et al., 1985; Mitz et al., 1991; Boussaoud and Wise, 1993a,b; Schall, 1997; Tehovnik et al., 2000; Koyama et al., 2004), the premotor visuomotor hand conditional activity in the human brain occurs anterior to the primary motor cortex hand representation, and the FEF occurs ventral and rostral to the premotor visuomotor hand conditional activity (Figs. 2-5). Our data indicate that the anatomo-functional relationships between the visuomotor hand conditional premotor-related activity and the saccadic eye movement activity are preserved from monkey to human (Fig. 5) and highlight the importance of detailed examination of the relationship of functional activity to local morphology on a subject-by- 
subject basis. The common practice of averaging a functional response across several brains and projecting the obtained activity peak in the average brain of the subjects studied can only provide an approximate location of the response, and any relationship of the functional activity to patterns of local morphological variation will be ignored. Thus, any relationship that the functional activity might have with local morphology will therefore not be revealed, and detailed functional organization will not be established.

\section{References}

Astafiev S, Shulman G, Stanley C, Snyder A, Van Essen D, Corbetta M (2003) Functional organization of human intraparietal and frontal cortex for attending, looking, and pointing. J Neurosci 23:4689-4699.

Blanke O, Spinelli L, Thut G, Michel CM, Perrig S, Landis T, Seeck M (2000) Location of the human frontal eye field as defined by electrical cortical stimulation: anatomical, functional and electrophysiological characteristics. NeuroReport 11:1907-1913.

Boettiger CA, D'Esposito M (2005) Frontal networks for learning and executing arbitrary stimulus-response associations. J Neurosci 25:2723-2732.

Boling W, Olivier A, Bittar RG, Reutens D (1999) Localization of hand motor activation in Broca's pli de passage moyen. J Neurosurg 91:903-910.

Boussaoud D, Wise SP (1993a) Primate frontal cortex: neuronal activity following attentional versus intentional cues. Exp Brain Res 95:15-27.

Boussaoud D, Wise SP (1993b) Primate frontal cortex: effects of stimulus and movement. Exp Brain Res 95:28-40.

Broca P (1888) Mémoires sur le cerveau de l'homme et des primates, pp 739-804. Paris: Reinwald.

Bruce CJ, Goldberg ME (1985) Primate frontal eye fields. I. Single neurons discharging before saccades. J Neurophysiol 53:603-635.

Bruce CJ, Goldberg ME, Bushnell MC, Stanton GB (1985) Primate frontal eye fields. II. Physiological and anatomical correlates of electrically evoked eye movements. J Neurophysiol 54:714-734.

Bullmore E, Brammer M, Williams SC, Rabe-Hesketh S, Janot N, David A, Mellers J, Howard R, Sham P (1996) Statistical methods of estimation and inference for functional MR image analysis. Magn Reson Med 35:261-277.

Collins DL, Neelin P, Peters TM, Evans AC (1994) Automatic 3D intersubject registration of MR volumetric data in standardized Talairach space. J Comput Assist Tomogr 18:192-205.

Corbetta M, Akbudak E, Conturo TE, Snyder AZ, Ollinger JM, Drury HA, Linenweber MR, Petersen SE, Raichle ME, Van Essen DC, Shulman GL (1998) A common network of functional areas for attention and eye movements. Neuron 21:761-773.

Cox RW, Jesmanowicz A (1999) Real-time 3D image registration for functional MRI. Magn Reson Med 42:1014-1018.

Crovitz HF, Zener K (1962) A group-test for assessing hand- and eyedominance. Am J Psychol 75:271-276.

Deiber MP, Wise SP, Honda M, Catalan MJ, Grafman J, Hallett M (1997) Frontal and parietal networks for conditional motor learning: a positron emission tomography study. J Neurophysiol 78:977-991.

Dum R, Strick P (2002) Motor areas in the frontal lobe of the primate. Physiol Behav 77:677-682.

Friston KJ, Holmes AP, Poline JB, Grasby PJ, Williams SC, Frackowiak RS, Turner R (1995) Analysis of fMRI time-series revisited. NeuroImage 2:45-53.

Gagnon D, O’Driscoll GA, Petrides M, Pike GB (2002) The effect of spatial and temporal information on saccades and neural activity in oculomotor structures. Brain 125:123-139.

Germain L, Lamarre Y (1993) Neuronal activity in the motor and premotor cortices before and after learning the associations between auditory stimuli and motor responses. Brain Res 611:175-179.

Germann J, Robbins S, Halsband U, Petrides M (2005) The precentral sulcal complex of the human brain: morphology and statistical probability maps. J Comp Neurol 493:334-356.

Glover GH (1999) Deconvolution of impulse response in event-related BOLD fMRI. NeuroImage 9:416-429.

Grafton ST, Fagg AH, Arbib MA (1998) Dorsal premotor cortex and conditional movement selection: a PET functional mapping study. J Neurophysiol 79:1092-1097.
Grosbras MH, Laird AR, Paus T (2005) Cortical regions involved in eye movements, shifts of attention, and gaze perception. Hum Brain Mapp 25:140-154.

Halsband U, Freund HJ (1990) Premotor cortex and conditional motor learning in man. Brain 113:207-222.

Halsband U, Passingham R (1982) The role of premotor and parietal cortex in the direction of action. Brain Res 240:368-372.

Johansen-Berg H, Rushworth MFS, Bogdanovic MD, Kischka U, Wimalaratna S, Matthews PM (2002) The role of ipsilateral premotor cortex in hand movement after stroke. Proc Natl Acad Sci USA 99:14518-14523.

Koyama M, Hasegawa I, Osada T, Adachi Y, Nakahara K, Miyashita Y (2004) Functional magnetic resonance imaging of macaque monkeys performing visually guided saccade tasks: comparison of cortical eye fields with humans. Neuron 41:795-807.

Kurata K, Tsuji T, Naraki S, Seino M, Abe Y (2000) Activation of the dorsal premotor cortex and pre-supplementary motor area of humans during an auditory conditional motor task. J Neurophysiol 84:1667-1672.

Luna B, Thulborn KR, Strojwas MH, McCurtain BJ, Berman RA, Genovese CR, Sweeney JA (1998) Dorsal cortical regions subserving visually guided saccades in humans: an fMRI study. Cereb Cortex 8:40-47.

Mitz AR, Godschalk M, Wise SP (1991) Learning-dependent neuronal activity in the premotor cortex: activity during the acquisition of conditional motor associations. J Neurosci 11:1855-1872.

Paus T (1996) Location and function of the human frontal eye-field: a selective review. Neuropsychologia 34:475-483.

Petit L, Haxby JV (1999) Functional anatomy of pursuit eye movements in humans as revealed by fMRI. J Neurophysiol 82:463-471.

Petit L, Clark VP, Ingeholm J, Haxby JV (1997) Dissociation of saccaderelated and pursuit-related activation in human frontal eye fields as revealed by fMRI. J Neurophysiol 77:3386-3390.

Petrides M (1982) Motor conditional associative-learning after selective prefrontal lesions in the monkey. Behav Brain Res 5:407-413.

Petrides M (1985) Deficits on conditional associative-learning tasks after frontal- and temporal-lobe lesions in man. Neuropsychologia 23:601-614.

Petrides M (1986) The effect of periarcuate lesions in the monkey on the performance of symmetrically and asymmetrically reinforced visual and auditory go, no-go tasks. J Neurosci 6:2054-2063.

Petrides M (1997) Visuo-motor conditional associative learning after frontal and temporal lesions in the human brain. Neuropsychologia 35:989-997.

Petrides M (2005) The rostral-caudal axis of cognitive control within the lateral frontal cortex. In: From monkey to human brain (Dehaene S, Duhamel J-R, Hauser MD, Rizzolatti G, eds), pp 293-314. Cambridge, MA: MIT.

Rizzolatti G, Luppino G (2001) The cortical motor system. Neuron 31:889-901.

Schall JD (1997) Visuomotor areas of the frontal lobe. In: Cerebral cortex (Rockland KS, Kaas JH, Peters A, eds), pp 527-638. New York: Plenum.

Schluter ND, Rushworth MFS, Passingham RE, Mills KR (1998) Temporary interference in human lateral premotor cortex suggests dominance for the selection of movements. A study using transcranial magnetic stimulation. Brain 121:785-799.

Talairach J, Tournoux P (1988) Co-planar stereotactic atlas of the human brain: 3-dimentional proportional system: an approach to cerebral imaging. Stuttgart, Germany: Thieme.

Tehovnik EJ, Sommer MA, Chou IH, Slocum WM, Schiller PH (2000) Eye fields in the frontal lobes of primates. Brain Res Brain Res Rev 32:413-448.

Toni I, Schluter ND, Josephs O, Friston K, Passingham RE (1999) Signal-, set- and movement-related activity in the human brain: an event-related fMRI study. Cereb Cortex 9:35-49.

Worsley KJ, Marett S, Vandal AC, Friston KJ, Evans AC (1996) A unified statistical approach for determining significant signals in images of cerebral activation. Hum Brain Mapp 4:58-73.

Worsley KJ, Liao CH, Aston J, Petre V, Duncan GH, Morales F, Evans AC (2002) A general statistical analysis for fMRI data. NeuroImage 15:1-15.

Yousry TA, Schmid UD, Alkadhi H, Schmidt D, Peraud A, Buettner A, Winkler P (1997) Localization of the motor hand area to a knob on the precentral gyrus-a new landmark. Brain 120:141-157. 